Conclusions A valid tool was developed that measured the baseline performance of the MMUH clinical pharmacy service for the safe prescribing of intermittent medicines. Clarification of the clinical pharmacy services SOP will lead to improved performance as pharmacists had varying interpretations of the SOP.

No conflict of interest.

\section{CPC-138 THE PRESCRIPTION OF ANTHRACYCLINES DURING PREGNANCY IN HAEMATOLOGY: CASE REPORTS AND LITERATURE REVIEW}

doi:10.1136/ejhpharm-2013-000276.595

${ }^{1} \mathrm{C}$ Peloso, ${ }^{1} \mathrm{MT}$ Baylatry, ${ }^{2} \mathrm{E}$ Elefant, ${ }^{3} \mathrm{~F}$ Isnard, ${ }^{1} \mathrm{C}$ Fernandez, ${ }^{1} \mathrm{AC}$ Joly. ${ }^{1}$ Saint-Antoine Hospital (APHP), Pharmacy, Paris, France; ${ }^{2}$ Trousseau Hospital (APHP), Crat, Paris, France: ${ }^{3}$ Saint-Antoine Hospital (APHP), Haematology, Paris, France

Background Anthracyclines are one of the most important groups of drugs used nowadays in cancer chemotherapy. Chemotherapy is essential in the management of haematological malignancies (HM). When acute leukaemia (AL), aggressive non-Hodgkin's lymphoma (NHL) or Hodgkin's lymphoma (HL) occur during pregnancy, chemotherapy is an emergency but foetal risk must be considered.

Purpose To evaluate foetal and maternal outcomes associated with the prescription of anthracyclines in pregnant women with HM.

Materials and Methods Cases of pregnant women with AL, NHL or HL treated by anthracyclines were collected from the Teratogenic Agent Information Centre (TAIC), a French reference centre providing specialised information for clinicians about drug use in pregnancy. A literature review was performed in the PubMed and Embase databases until May 2012 (keywords: pregnancy, acute leukaemia, Hodgkin lymphoma, non-Hodgkin lymphoma, cancer chemotherapy, doxorubicin, daunorubicin and idarubicin). Selection criteria of articles: diagnosis of HM and anthracycline prescription during pregnancy, foetal outcome.

Results We report 5 cases of pregnant women with HM (4 AL, $1 \mathrm{HL}$ ) treated early in the 3rd trimester by chemotherapy with doxorubicin or daunorubicin at standard dosage. All 5 newborns were normal, but 2 were premature deliveries. 3 maternal outcomes were complete remission (2 unknown). 81 articles were selected, corresponding to 134 pregnant women with AL (95 cases), HL (16) or NHL (23) treated by chemotherapy with daunorubicin (65 cases), doxorubicin (59) or idarubicin (10). Normal neonatal outcomes $(100 / 134)$ were $88 \%, 68 \%$ and $40 \%$ for doxorubicin, daunorubicin and idarubicin respectively, 79\%, 77\% and $45 \%$ for exposure from $3 \mathrm{rd}$ (26 cases), 2 nd (69) and 1st trimester (11) respectively and $96 \%, 81 \%$ and $68 \%$ in $\mathrm{NHL}, \mathrm{LH}$ and $\mathrm{AL}$ respectively. Foetal toxicities were death (20), growth retardation (8) and congenital abnormalities (6). Only idarubicin was associated with foetal cardiomyopathy. 97 maternal outcomes were known with remissions (71 cases) and progressions, relapses or deaths (26 cases).

Conclusions Embryo-foetal toxicity depends on gestational age, anthracycline and HM. 2nd or 3rd trimester exposures were mainly associated with favourable neonatal outcomes. Idarubicin was specifically associated with a risk of foetal cardiotoxicity, probably due to its lipophilic nature, facilitating placental transfer. Unfavourable foetal outcomes were more frequent in AL compared to lymphomas, probably reflecting that chemotherapy can never be delayed till post-partum in AL. It is possible to prescribe anthracyclines for HM in the 2nd and 3rd trimesters of pregnancy with minimal risk to the developing foetus but then the treatment must be conducted by a multidisciplinary team.

No conflict of interest.

\section{CPC-139 THERAPEUTIC DRUG MONITORING FOR GLYCOPEPTIDES AND AMINOGLYCOSIDES: ACTUAL SITUATION AND PERSPECTIVES IN A FRENCH UNIVERSITY HOSPITAL}

doi:10.1136/ejhpharm-2013-000276.596

'LA Arnoux, 'E Boschetti, ${ }^{2}$ S Bevilacqua, II May, 'B Demore. 'Hopital Brabois Chu de Nancy, Pharmacie Brabois, Vandoeuvre Les Nancy, France; ${ }^{2}$ Hopital Brabois Chu de NANCY, Service des Maladies Infectieuses et Tropicales, Vandoeuvre Les Nancy, France

Background Optimising glycopeptide and aminoglycoside treatment with Therapeutic Drug Monitoring is recommended. Underdosing can lead to resistance and ineffective treatment while over-dosing is associated with toxicity.

Purpose To evaluate current practise by monitoring aminoglycosides and glycopeptides in a French university hospital: levels (trough and peak concentrations) and percentage of optimal concentrations based on our internal antibiotics guide.

Materials and Methods Prescriptions for glycopeptides and/or aminoglycosides, of which at least one dose had been given, were reviewed over one month (February-March 2012). Our data pool contained: patient characteristics, infection and antibiotic treatment background, serum concentration.

Results A wide range of official optimal target serum concentrations has been recommended (Consensus Review of the American Society of Health-System Pharmacists, French Pharmacology and Therapeutic Society, internal guidelines, etc.)

91 prescriptions (31 aminoglycosides, 60 glycopeptides) were analysed: the largest percentage was represented by vancomycin $(55 \%) 80 \%$ of which were for continuous infusion. Serum vancomycin concentrations are optimised by using continuous regimens (Table 1).

For the two regimens, (continuous and intermittent, 10\% of trough vancomycin serum concentrations were below $10 \mathrm{mg} / \mathrm{L}$, exposing the patient to to subtherapeutic doses and a higher risk of selecting resistant microorganisms.

10 prescriptions for teicoplanin were reviewed: $70 \%$ of serum concentrations were below $20 \mathrm{mg} / \mathrm{L}$ and $30 \%$ below $10 \mathrm{mg} / \mathrm{L}$.

$50 \%$ of aminoglycosides trough concentrations were below the internal guideline values and target peak concentrations were not reached (amikacin: 67\% under $60 \mathrm{mg} / \mathrm{L}$, gentamycin: 90\% under $30 \mathrm{mg} / \mathrm{L}$ ).

Conclusions Most aminoglycosides and glycopeptides concentrations didn't reach required therapeutic levels during this study. Consensus guidelines should be proposed to avoid bacterial resistance and guide clinical practise.

Abstract CPC-139 Table 1 Serum vancomycin concentrations vary with the infusion regimens

\begin{tabular}{llll}
\hline & & $\begin{array}{l}\text { Continuous infusion } \\
\text { regimens }\end{array}$ & $\begin{array}{l}\text { Intermittent infusion } \\
\text { regimens }\end{array}$ \\
\hline $\begin{array}{l}\text { Optimal vancomycin } \\
\text { concentrations }\end{array}$ & {$[20-30] \mathrm{mg} / \mathrm{L}$} & $42 \%$ & $27 \%$ \\
$\begin{array}{c}\text { Subtherapeutic vancomycin } \\
\text { concentrations }\end{array}$ & $<20 \mathrm{mg} / \mathrm{L}$ & $33 \%$ & $54 \%$ \\
& $<10 \mathrm{mg} / \mathrm{L}$ & $8 \%$ & $27 \%$ \\
\hline
\end{tabular}

No conflict of interest.

\section{CPC-140 THERAPEUTIC OPTIONS IN ANTI-NMDA RECEPTOR ENCEPHALITIS}

doi:10.1136/ejhpharm-2013-000276.597

A Madrid Paredes, R López Sepúlveda, C García Collado, N Martínez Casanova, E Puerta García, B Cancela Díez, MA Calleja Hernández. Virgen de las Nieves University Hospital, Pharmacy, Granada, Spain 